\title{
A mouse prostate cancer model induced by Hedgehog overexpression
}

\author{
Bo-Yie Chen ${ }^{1, \dagger}$, David Pei-Cheng Lin ${ }^{1,2, \dagger}$, Jer-Yuh Liu ${ }^{1}$, Han Chang ${ }^{3}$, Po-Hsuan \\ Huang ${ }^{4}$, Yie-Ling Chen ${ }^{5} \&$ Han-Hsin Chang ${ }^{5, *}$ \\ ${ }^{1}$ Institute of Biochemistry and Biotechnology, Chung Shan Medical University, Taichung, Taiwan; ${ }^{2}$ School of \\ Medical Laboratory and Biotechnology, Chung Shan Medical University, Taichung, Taiwan; ${ }^{3}$ Department of \\ Pathology, Chung Shan Medical University, Taichung, Taiwan; ${ }^{4}$ Department of Urology, Chung Shan \\ Medical University, Taichung, Taiwan; ${ }^{5}$ Institute of Nutrition, Chung Shan Medical University, Taichung, \\ Taiwan
}

Received 16 May 2005; accepted 28 November 2005

(C) 2005 National Science Council, Taipei

Key words: animal model, Hedgehog, prostate cancer, tumorigenesis

\section{Summary}

Hedgehog is a regulatory protein during embryonic development and its abnormal activation in adult tissues has been implicated in tumorigenesis within sites where epithelial-mesenchymal interactions take place. In the prostate, Hedgehog signaling activation was observed during advanced cancer progression and metastasis, but whether Hedgehog overexpression can initiate prostate tumorigenesis remains unknown. We introduced a Hedgehog-expressing vector by intra-prostate injection and electroporation to address the effects of Hedgehog overexpression. The manipulation caused lesions with characteristic prostatic intraepithelial neoplasia or even prostatic cancer $(\mathrm{CaP})$ phenotypes within 30 days, with Hedgehog overexpression demonstrated by immunohistochemistry and Western blot detections. The tumorigenic phenotypes were confirmed by discontinuity of basal cell marker p63, mix-up of CK-8/CK-18 positive epithelial cells in the stoma as well as absence of $\alpha$-SMA positive fibro-muscular sheath. Comparable Hedgehog overexpression was found in human $\mathrm{CaP}$ specimen. Thus, Hedgehog overexpression induced prostate tumorigenesis starting from the normal status. Furthermore, a mouse prostate cancer model induced by Hedgehog overexpression was established and may be used for testing novel therapeutical approaches targeting at Hedgehog signaling pathway.

\section{Introduction}

Prostate cancer, apart from skin cancer, is the most common male malignancy and the second leading cause of cancer deaths in men in the United States [1]. It had surpassed lung cancer in 1990 and was estimated to cause 31,000 deaths in 2002 in the United States alone [2]. Despite the increasing incidence, prostate cancer presents

\footnotetext{
*To whom correspondence should be addressed. Fax: + 886-42324-8175; E-mail: jhhc@csmu.edu.tw

${ }^{\dagger}$ These authors have contributed equally to this work.
}

some obstacles that hind clinicians and basic researchers from understanding its pathogenesis. It is characterized by slow clinical progression, involvement of multiple genetic and epigenetic events, multifocal and heterogeneous nature of tumorigenesis, and difficulty in prognosis of disease progression [3, 4]. Given these difficulties, mouse models are advantageous for studying prostate cancer, despite intrinsically anatomical differences and probably different molecular mechanisms underlying prostate carcinogenesis [3, 5].

Hedgehog signaling has been reported to involve in many processes during embryogenesis, 
including dorsoventral patterning of body axis, specification of neuronal and oligodendrocytic cell fate, axonal outgrowth, cell proliferation, cell differentiation, and cell survival [6, 7]. Recent studies showed a key role of Shh signaling in mediating endoderm-derived tissue formation, including prostate formation. Shh was expressed during prostatic branching morphogenesis, suggesting involvement in the initiation of androgendependent prostate development [8, 9]. Mutant analyses revealed a critical role of Shh signaling, but Shh expression appeared not regulated by androgens [10, 11]. Androgen supplements was shown to reverse Shh mutant phenotypes [11], indicating that the role of Shh could be replaced by androgens during prostate development.

Like many developmentally critical genes, Hedgehog has been shown to involve in tumorigenesis. Abnormal Shh hyperactivation was found in small-cell lung cancer [12], pancreatic cancer [13], digestive tract tumors [14], and ameloblastomas [15]. Mutations in Ptc gene, a tumor suppressor gene and a Shh signaling pathway repressor, was shown to cause cerebellar medulloblastomas [16] and basal cell carcinomas [17] in mice, and was implicated in human superficial bladder cancer [18]. In concordance with the observations in other tumors, several lines of evidences have indicated Hedgehog signaling in prostate tumorigenesis. First, SHH pathway components, for example GLI, were detected in adult human prostate cancer, often with enhanced levels in tumors as compared to those in the normal status [19-21]. Second, a xenograft model was used to elucidate paracrine interactions between Shh-expressing human LNCaP tumor cells and host mouse stromal cells [22]. The LNCaP cells were genetically engineered to express Shh at high levels that dramatically accelerate tumor growth with increased Gli expression when co-injected with Matrigel subcutaneously. Third, prostate cancer cell lines, including PC3, LNCaP, DU145, 22RV1, and TSU, were shown to express high levels of Hedgehog or its target genes [20, 21, 23]. When Hedgehog pathway was blocked with either Smo antagonist, cyclopamine, or GLI-1 RNA interference, apoptosis was induced and cell proliferation or invasiveness were inhibited [20, 21, 23]. Fourth, Hedgehog signaling modulated metastatic potential of rodent prostate cancer cell lines [23]. Fifth, when PrE cells, a putative prostate epithelium progenitor cell line, were stably transfected for GLI expression, the cells exhibited unlimited growth in vitro and formed aggressive subcutaneous tumors after transplantation into an athymic mouse [23].

Despite these previously published data, there is so far no mouse prostate cancer model caused initially by in vivo Hedgehog dysregulation from a normal status and in the prostate itself. Hence, a potential role of Hedgehog in the initiation of prostate cancer remains to be elucidated. In this report, we addressed the effects of Hedgehog overexpression by introducing directly a Hedgehogexpressing vector into normal prostates.

\section{Materials and methods}

\section{Plasmids}

Mouse Shh-expressing and vehicle vectors, pCX-shh-IG and pCX-IG, were kindly provided by Dr Kerby C. Oberg, Loma Linda University [24]. The pCX-shh-IG contained a Shh insert tagged with green fluorescence protein (GFP) sequence driven by a CMV promoter. The vehicle control pCX-IG contained the same CMV promoter and the GFP tag, but without the Shh insert. Therefore, presence of GFP in prostates indicated successful introduction of pCX-shh-IG vector and expression of Hedgehog protein.

\section{Injection and electroporation}

FVB strain mice aged 8-10 weeks were purchased from National Laboratory Animal Center, Academia Sinica, Taipei for use in the injections. The mice were anesthetized with phenobarbital and exposed of their prostate glands by surgery. For each injection, $10 \mu \mathrm{l}$ of pCX-shh-IG at $0.1 \mu \mathrm{g} / \mu \mathrm{l}$ in $0.9 \%$ $\mathrm{NaCl}(n=25)$ was injected into the anterior prostate lobe or dorsolateral lobe or both (Figure 1a). Parallel injections with $10 \mu \mathrm{l}$ of pCX-IG in $0.9 \%$ $\mathrm{NaCl}(n=10)$ or $0.9 \% \mathrm{NaCl}(n=10)$ were performed as vehicle or blank controls. Since Hedgehog had been known as a secretory protein [6], the expressed protein was assumed to disperse freely within the prostate. We performed 1 or 2 injections at random sites in each prostate lobe. After injections, the prostate lobes were subjected to electric pulses for 10 s set at 20 volts, $1-2$ pulses per second, using a Digitimer DS7 Stimulator (Digitimer, Hertfordshire, England). The injection and 
(a)

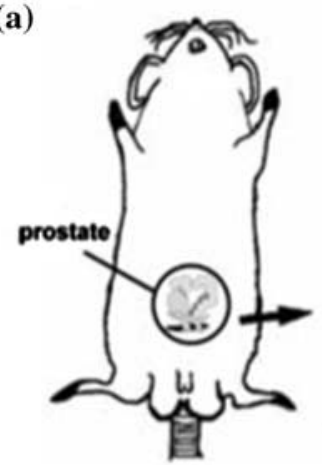

(b)

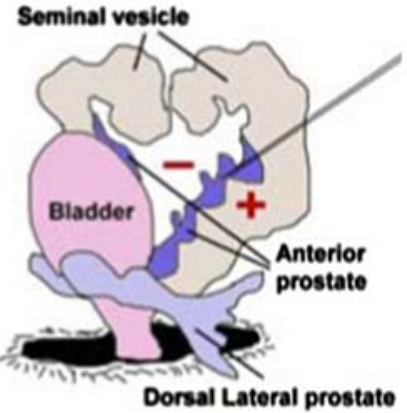

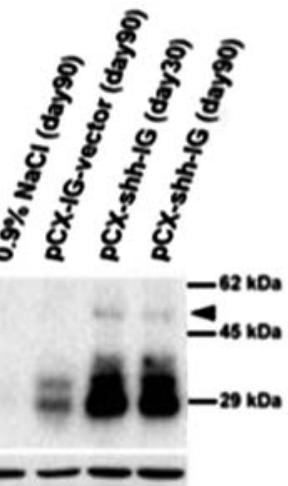
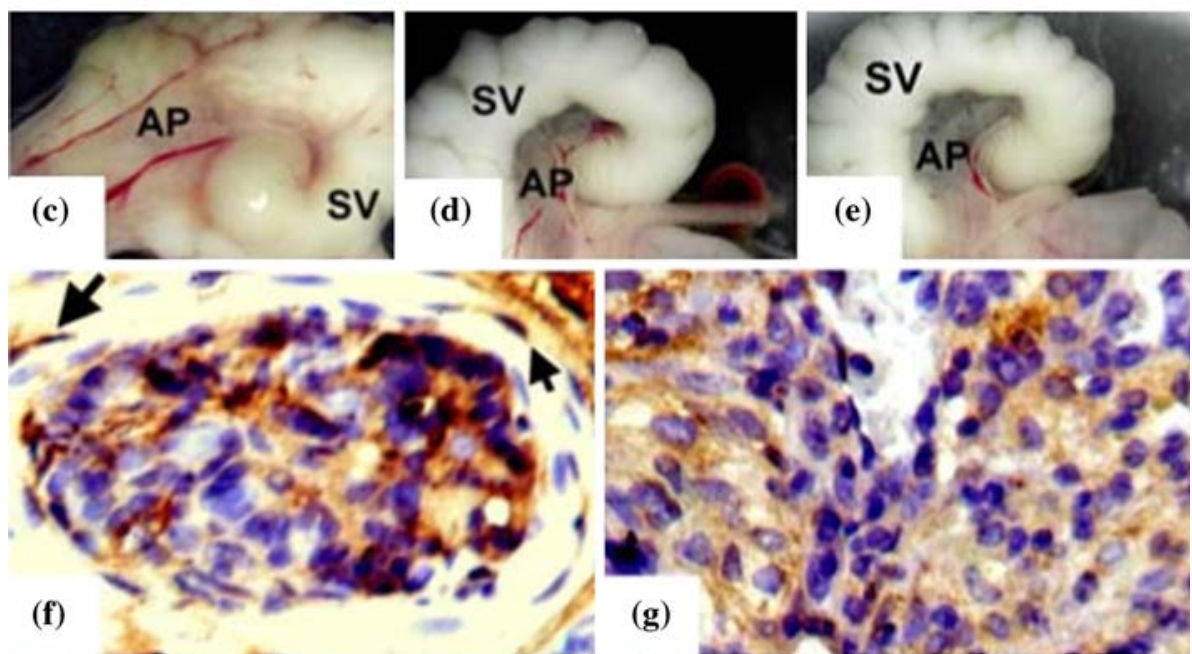

(g)

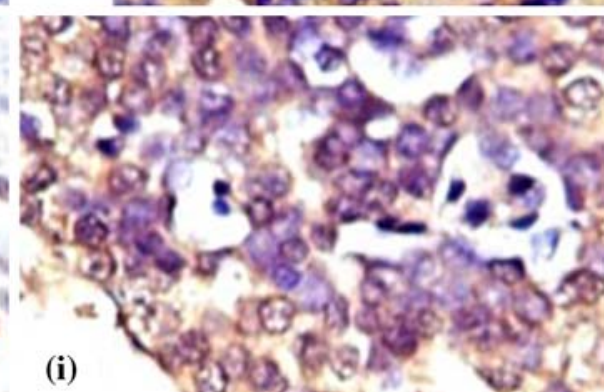

(h)

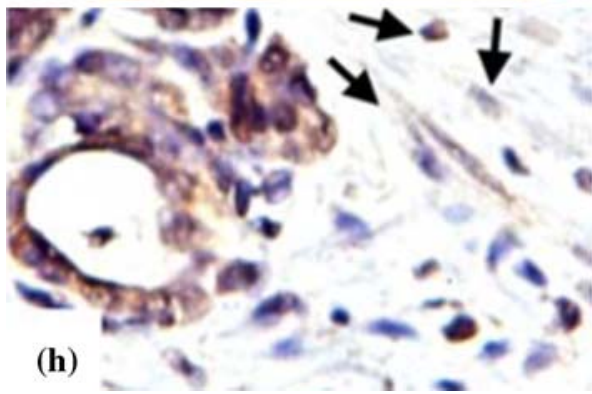

(i)

Figure 1. Schematic representation of vector injection and electroporation, confirmation of Hedgehog overexpression and its expression patterns, and macro- and microscopic effects of Hedgehog overexpression. (a) The mouse prostate was exposed by surgery and injected through a glass needle with $10 \mu \mathrm{l}$ of pCX-Shh-IG or pCX-IG at $0.1 \mu \mathrm{g} / \mu \mathrm{l}$ in $0.9 \% \mathrm{NaCl}$, or $0.9 \% \mathrm{NaCl}$ alone. After injections, the prostate lobes were subjected to electric pulses for $10 \mathrm{~s}$ set at 20 volts, $1-2$ pulses per second; the mice were then stitched, recovered, and maintained until dissection. (b) Western blot analyses confirmed overexpression of Hedgehog protein tagged with GFP (indicated by arrowhead) that persisted for 90 days after injection. (c) Evident overgrowth with more blood vessels but less lobular formation in the seminal vesicle, was seen in the pCX-shh-IG-injected mouse prostate, as compared to the $0.9 \% \mathrm{NaCl}$-injected control in (d) or the pCX-IG-injected control in (e). (f) and (g) Immunodetection of mouse Hedgehog expression using 5E1 antibody following pCX-shh-IG injection. The highly expressed Hedgehog protein was detected in the epithelium as well as in the stroma (indicated by arrows), comparable to that in the human CaP specimen using N-19 antibody for Hedgehog detection in (h) and (i). Magnifications: $20 \times$ for (c) to (e); $400 \times$ for (f) to (i).

electroporation protocol was according to Nakamura and Funahashi [25] with minor modifications as detailed here to suit its use for mouse prostates.
After the injection, the mice were allowed to recover and maintained until dissection at days 7, 20, 30, and 90 after the manipulation. 
Mouse PIN and CaP determination following Hedgehog overexpression

Mouse PIN and CaP were determined according to their architecture, differentiation pattern, and degree of cytological atypia. Antibodies against p63 (basal cell marker), CK-8/CK-18 (epithelial cell marker), and $\alpha$-SMA (fibro-muscular cell marker) were used to support confirmation of PIN, CaP, and invasive CaP status. PIN was identified with one or two layers of atypical cells exhibiting large, pleomorphic nuclei and prominent nucleoli [26]. PIN could reach a high grade status with large infolded glands surrounded by a discontinuous layer of basal cells. Cap was distinguished from PIN with smaller glands and in lack of basal cells, although it also contains atypical cells.

\section{Human tissues}

The human prostate tissues were obtained from 40 patients who had received radical prostectomy or needle biopsy at Department of Pathology, Chung Shan Medical University. The presence of $\mathrm{CaP}$ was confirmed with histopathological examinations performed by clinical pathologists. The Gleason gradings were $2+3 \quad(n=22), \quad 3+3$ $(n=14)$, and $4+4(n=4)$. All tissue procurement procedures and experiments were reviewed and approved by the Institutional Review Board of Chung Shan Medical University.

\section{Immunohistochemistry}

Tissues were dissected, fixed in 4\% paraformaldehyde in PBS (Sigma), and processed to obtain $5 \mu \mathrm{m}$ thick sections following standard histological preparations. For anti-Shh (5E1) and anti-GFP detections, the tissue sections were processed through citrate buffer ( $\mathrm{pH} 2.0$ ) for $4 \mathrm{~min}$, followed by another citrate buffer $(\mathrm{pH} 9.0)$ for $5 \mathrm{~min}$ in a microwave oven set at 750 Watts, and then thoroughly washed with Tris-buffered saline before antibody binding. Antibodies against p63, CK-8/ CK-18, and $\alpha$-SMA were used for confirmation of tumorigenic phenotypes and were processed following the same procedures as for anti-Shh and anti-GFP detections. For anti-SHH (N-19), the tissue sections were treated with citrate buffer (pH 6) for $10 \mathrm{~min}$ in a microwave oven set at
750 Watts before antibody binding. The anti-Shh was purchased from Developmental Studies Hybridoma Bank (Iowa City, IA, USA); antiSHH, anti-p63, anti-CK-8/CK-18, anti- $\alpha$-SMA, and anti-GFP from Santa Cruz Biotechnology Inc. (Santa Cruz, CA, USA). Peroxidase linked avidin/ biotin complex reagents and the ABC immunoperoxidase kits were used for signal detection and were purchased from Vector Laboratories (Burlingame, CA, USA). Parallel detections were performed as negative controls without antibody addition. Triple repeats were performed for each mouse and human specimen before positive results were regarded as genuine.

\section{Western analyses}

For Western analyses, the prostates were dissected and collected in microtubes containing $1 \mathrm{ml}$ of RIPA buffer (Gibco BRL, Gaithersburg, MD, USA) with freshly prepared $1 \mathrm{mM}$ PMSF and $5 \mathrm{mM}$ EDTA in PBS. The mixtures were centrifuged at $14,000 \times g$ for $10 \mathrm{~min}$ and the protein concentrations in the supernatants were determined by the modified Lowry method [27]. The preparations were resolved by SDS-PAGE on $12 \%$ polyacrylamide slab gels $(8 \mathrm{~cm} \times 7 \mathrm{~cm} \times 0.075 \mathrm{~cm})$ according to the method of Laemmli [28]. The proteins were blotted from the polyacrylamide gels onto Immobilon-P membranes (Amersham Biosciences, Piscataway, NJ, USA) in 5\% milk in TBST (0.05\% Tween-20, 20 mM Tris- $-\mathrm{HCl} \mathrm{pH} 7.4,0.9 \%$ $\mathrm{NaCl}$ ) and then incubated with anti-Shh, and together with anti- $\alpha$-tubulin as loading controls. The membranes were washed three times with TBST for $20 \mathrm{~min}$ prior to $1 \mathrm{~h}$ incubation with a goat antirabbit secondary antibody conjugated with horseradish peroxidase (Chemicon, Temecula, CA, USA) and then visualized using an enhanced chemiluminescent system (Boehringer-Mannhein, Mannhein, Germany).

\section{Results}

Confirmation of Hedgehog overexpression in the pCX-shh-IG injections

To solidify any data obtained as a result of Hedgehog overexpression, we examined Hedgehog 
expression status after the manipulation. We first examined the presence of GFP signals in wholemount preparations and in tissue sections. When both methods showed no convincing signal, Western analysis was used as a double check. In wholemounts (data not shown), GFP signals were detected in 15 prostates in a total of $25 \mathrm{pCX}$-shhIG injections and in 8 of the $10 \mathrm{pCX}-\mathrm{IG}$ injections, but in none of the $0.9 \% \mathrm{NaCl}$ blank controls. With immunohistochemistry, 23 of the 25 pCX-shh-IG injections and 8 of the 10 pCX-IG injections exhibited definite signals for GFP, with no positive signal detected in the $0.9 \% \mathrm{NaCl}$ injections (Table 1). The two pCX-shh-IG injections without definite GFP signal in tissue sections were doublechecked using Western analyses (Figure 1b) and RT-PCR (data not shown); both were found positive for GFP. We then checked by immunohistochemistry using anti-Shh and confirmed that all pCX-shh-IG injections positive for GFP were also evidently positive for Hedgehog protein expression, whereas no comparable signal was detected in the pCX-IG vehicle controls or the $0.9 \% \mathrm{NaCl}$ blank controls. Therefore, the present data showed $100 \%(25 / 25)$ efficiency in introducing a Hedgehog overexpression status in the pCXshh-IG-injected prostates. Furthermore, the data demonstrated sustaining Hedgehog expression up to 90 days after the manipulation (Table 1 and Figure 1b).

Gross morphological effects with Hedgehog overexpression in the prostates

Gross morphological effects were examined at days 7, 20,30, and 90 after the manipulation and the most prominent changes were found at day 30 so far. By day 30 after the injection, the anterior prostates (AP) of the pCX-shh-IG injections exhibited overgrowth (Figure 1c), in contrast to those seen in the pCX-IG vehicle controls (Figure 1d) or in the $0.9 \% \mathrm{NaCl}$ blank controls (Figure 1e). We assumed that the overgrowth in the pCX-shh-IG-injected prostates was due to the effects of Hedgehog overexpression, since no comparable result was found in the two control groups. In addition, seminal vesicles (SV) in the pCX-shh-IG-injected prostates showed less evident lobular formation as compared to those in the pCX-IG and the $0.9 \% \mathrm{NaCl}$ injections (Figure 1c vs. Figure $1 \mathrm{~d}$ and e). The size of seminal vesicles appeared to enlarge with pCX-shh-IG injections. Furthermore, hypervascularization in the prostates was observed in wholemount preparations Figure 1c) as well as in tissue sections (Figure 2d).

\section{Mouse Hedgehog overexpression patterns were comparable to human conditions}

Conceivably, any animal model should be reflective of human conditions so that data from animal analyses could be applicable to human diseases. To solidify that the mouse prostates with Hedgehog overexpression could be used as study models for the human conditions, we compared the $\mathrm{pCX}$ shh-IG-injected mouse prostates with the human $\mathrm{CaP}$ specimen in their Hedgehog expression patterns by immunohistochemistry. Activation of Hedgehog was seen in 38 out of the 40 human specimen (Figure $1 \mathrm{~h}$ and $\mathrm{i}$ ), being in both the epithelial and the stromal cells, and the expression patterns were comparable to those observed in the mouse (Figure 1f and g). The comparable Hedgehog overexpression patterns in the mouse indicated phenocopying of the human status.

\section{PIN and CaP formation with Hedgehog overexpression}

The overgrowth with Hedgehog overexpression was further analyzed microscopically to elucidate whether prostate tumorigenesis had occurred. Prostatic intraepithelial neoplasia (PIN) was found in all pCX-shh-IG-injected prostates $(25 / 25)$ at 7 , 20, 30 and 90 days after pCX-shh-IG injections (Table 1; Figures 1f and g, 2c, f-h). Unlike the conditions in the $0.9 \% \mathrm{NaCl}$-injected (Figure 2a) and the pCX-IG-injected controls (Figure 2b), the pCX-shh-IG-injected prostate tissue sections showed infolded glands with stromal hyperplasia (Figure 2c) and often also with hypervascularization (Figure 2d). Derangements of epithelial cells were found in the pCX-shh-IG-injected prostates (Figures $1 \mathrm{f}$ and $\mathrm{g}, 2 \mathrm{f}-\mathrm{h}$ ), but not in the controls (Figure 2e). The derangements and infoldings, we believe, were not due to artifacts of oblique slicing, since the atypical cells were often seen in multifocal sites and were heterogeneous to surrounding typical epithelial glands within a same tissue (Figure 2c). 


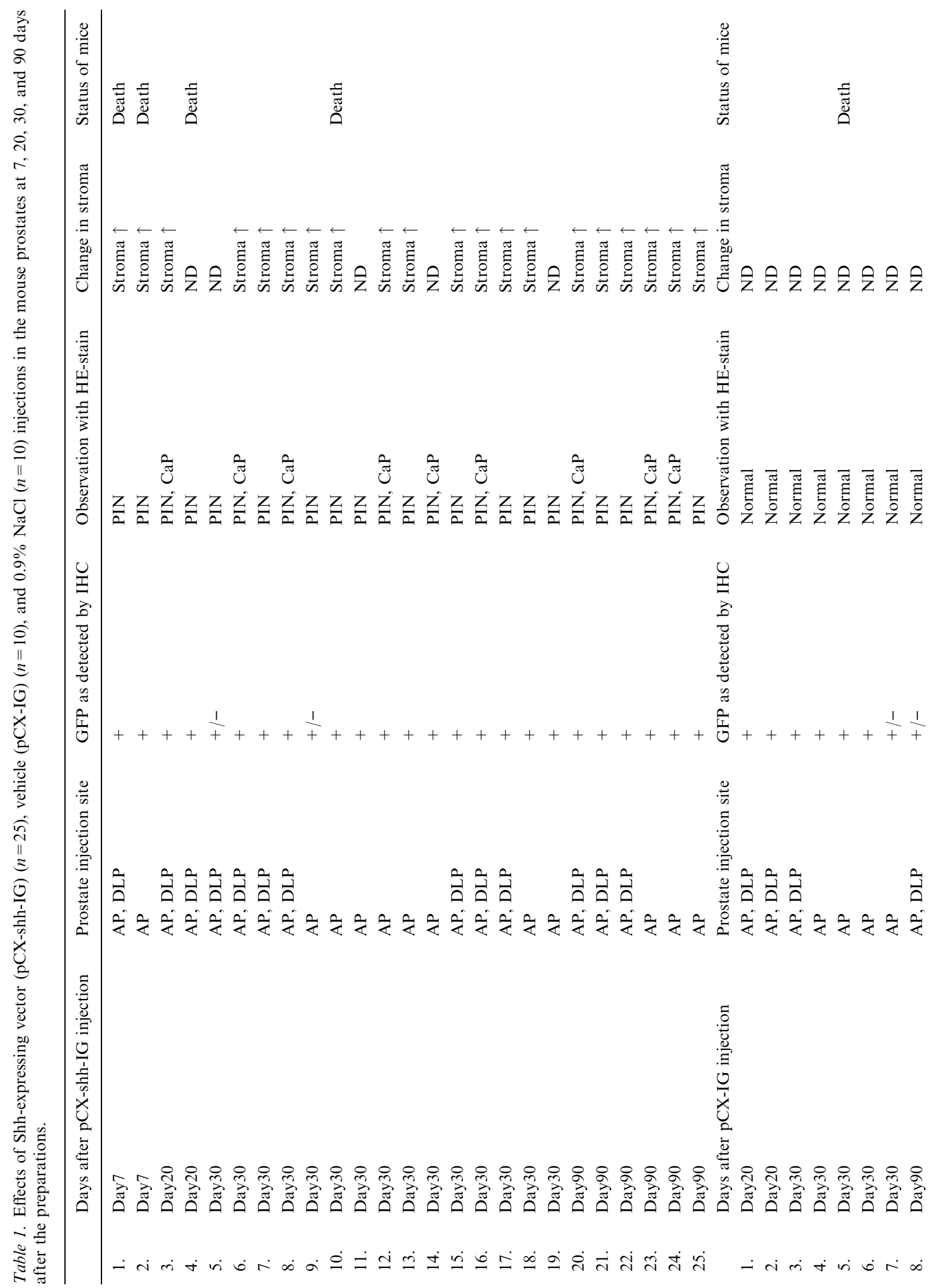




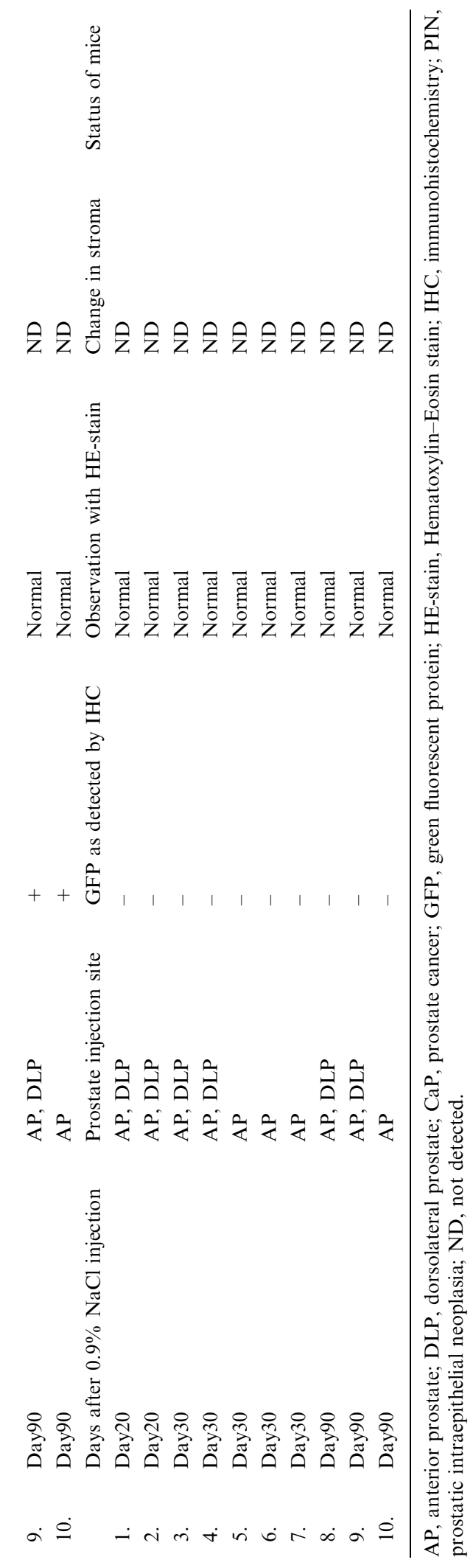

For the same reason, we believe that the observed abnormalities were not due to non-specific acute inflammatory responses to the injection and electroporation procedures. These heterogeneous and multifocal characteristics also indicated phenocopying of the human tumorigenesis.

In humans, high grade PIN is the believed precursor of $\mathrm{CaP}$. Both high grade PIN and $\mathrm{CaP}$, compared to normal prostate glands, have enlarged nuclei with prominent nucleoli. PIN is characterized by large infolded glands surrounded by a discontinuous layer of basal cells, whereas $\mathrm{CaP}$ glands are smaller and lack basal cells. To confirm PIN and CaP formation in the present mouse Hedgehog overexpression model, these microscopic phenotypes were examined. With higher magnification, the epithelial cells with Hedgehog overexpression showed enlarged nuclei with prominent nucleoli, typical of high grade PIN (Figure 3b) and $\mathrm{CaP}$ (Figure $3 \mathrm{c}$ and d) formations; these atypical cells were not found in the $0.9 \% \mathrm{NaCl}$ blank controls or the pCX-IG vehicle controls (Figure 3a). Further indications of PIN formation, as it was distinguished from $\mathrm{CaP}$, were the loss of basal cell continuity confirmed by p63 immunodetection (Figure $4 \mathrm{a}$ vs. b). It appeared that the basal cells began to lose their immunoreactivity to p63 antibody as the epithelial cells became invasive (Figure 4c). When $\mathrm{CaP}$ was formed, no evident p63 immunoreactivity was detected (Figure 4d). The invasive $\mathrm{CaP}$ formation was supported by invasion of $\mathrm{CK}-8 / \mathrm{CK}-18$ positive epithelial cells into the stroma (Figure 4f), in contrast to the normal glandular positioning in the controls (Figure 4e). We also found thinner and disrupted distribution of fibro-muscular cell marker $\alpha$-SMA in the pCX-shh-IG-injected prostates (Figure 4h), indicating muscle layer disruption and epithelial invasion. This disruption of fibromuscular sheath was not found in the controls injected with $0.9 \% \mathrm{NaCl}$ (Figure $4 \mathrm{~g}$ ).

Based on these criteria, $\mathrm{CaP}$ formation was observed in 9 cases $(9 / 25)$ after pCX-shh-IG injections (Table 1). All of the observed PIN and $\mathrm{CaP}$ formations were not found in the pCX-IG vehicle $(0 / 10)$, nor in the $0.9 \% \mathrm{NaCl}$ controls $(0 /$ 10). These results indicated that the characteristics of PIN and $\mathrm{CaP}$ formation were specifically due to Hedgehog overexpression, instead of the procedures of injections or electroporations. 

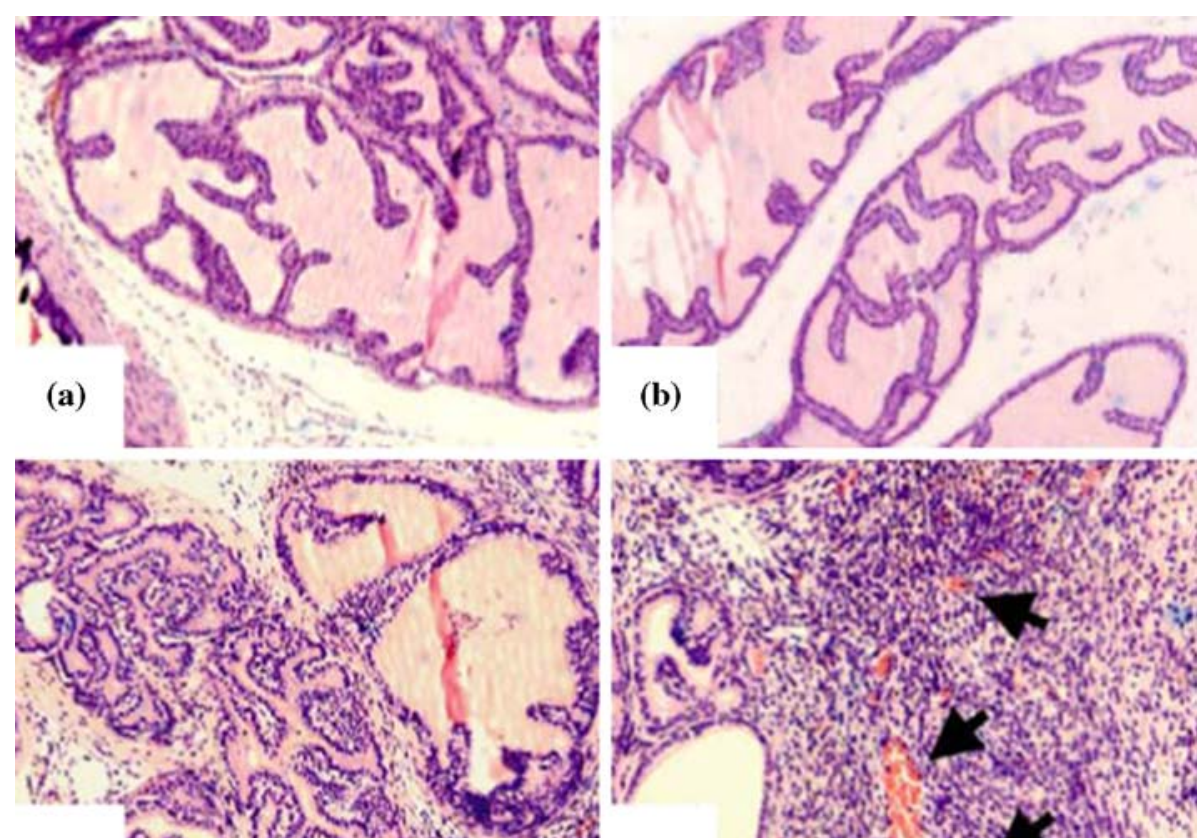

(c)
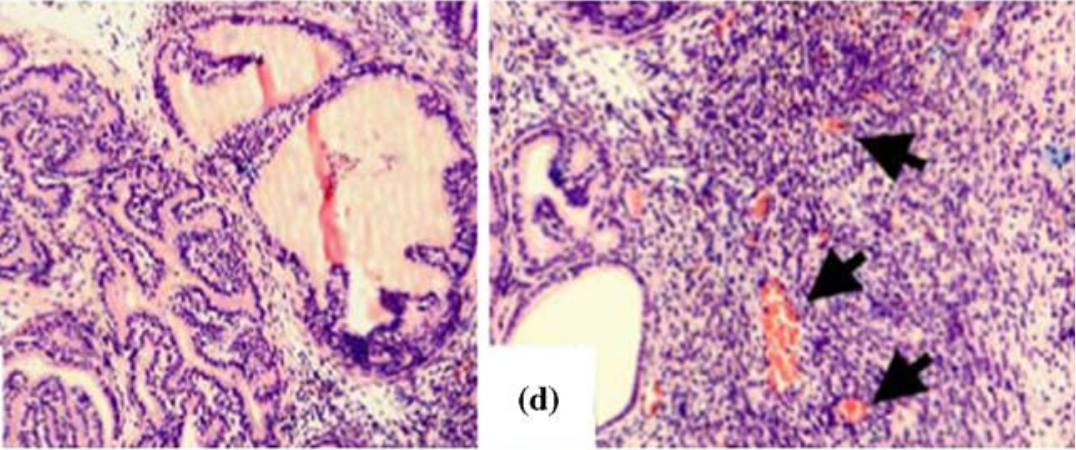

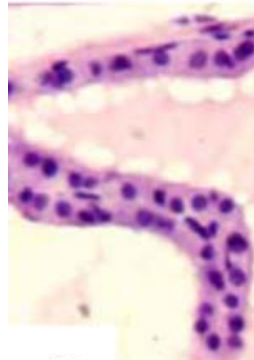

(e)
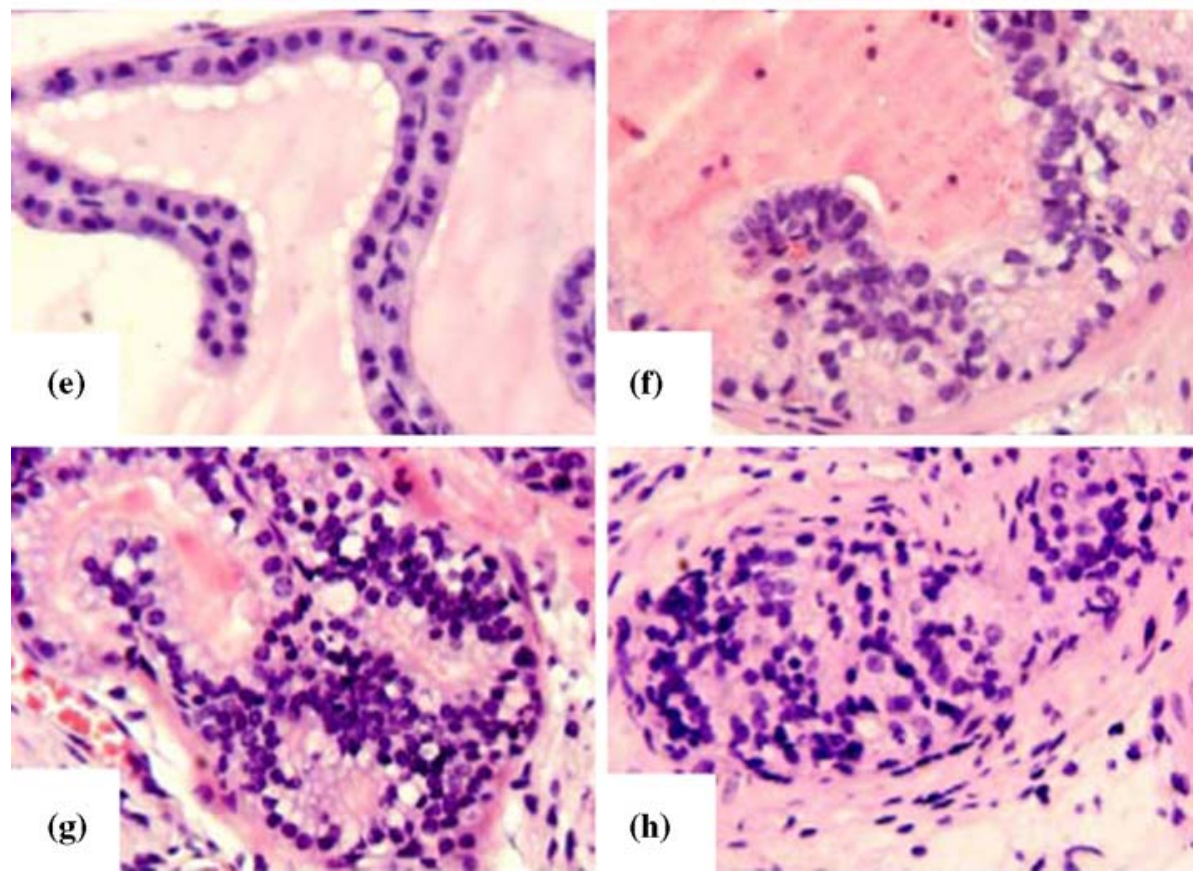

Figure 2. Histological effects of Hedgehog overexpression at 30 days after the injection as shown by Hematoxylin-Eosin stain. Prostates with $0.9 \% \mathrm{NaCl}$ injection in (a) and pCX-IG injection in (b) showed no tumorigenic characteristics. (c) A pCX-shh-IGinjected prostate showed glandular infoldings and stromal hyperplasia. (d) A pCX-shh-IG-injected prostate showed stromal hyperplasia and hypervascularization (indicated by arrows). (e) A pCX-IG-injected prostate showed normal features of epithelial and basal cells. (f-h) pCX-shh-IG-injected prostates showed different levels of PIN formation. Few layers of atypical cells were found in (f), but more layers were deployed in $(\mathrm{g})$ and even more in (h). Magnifications: $100 \times$ for (a) to (d); $400 \times$ for (e) to (h). 


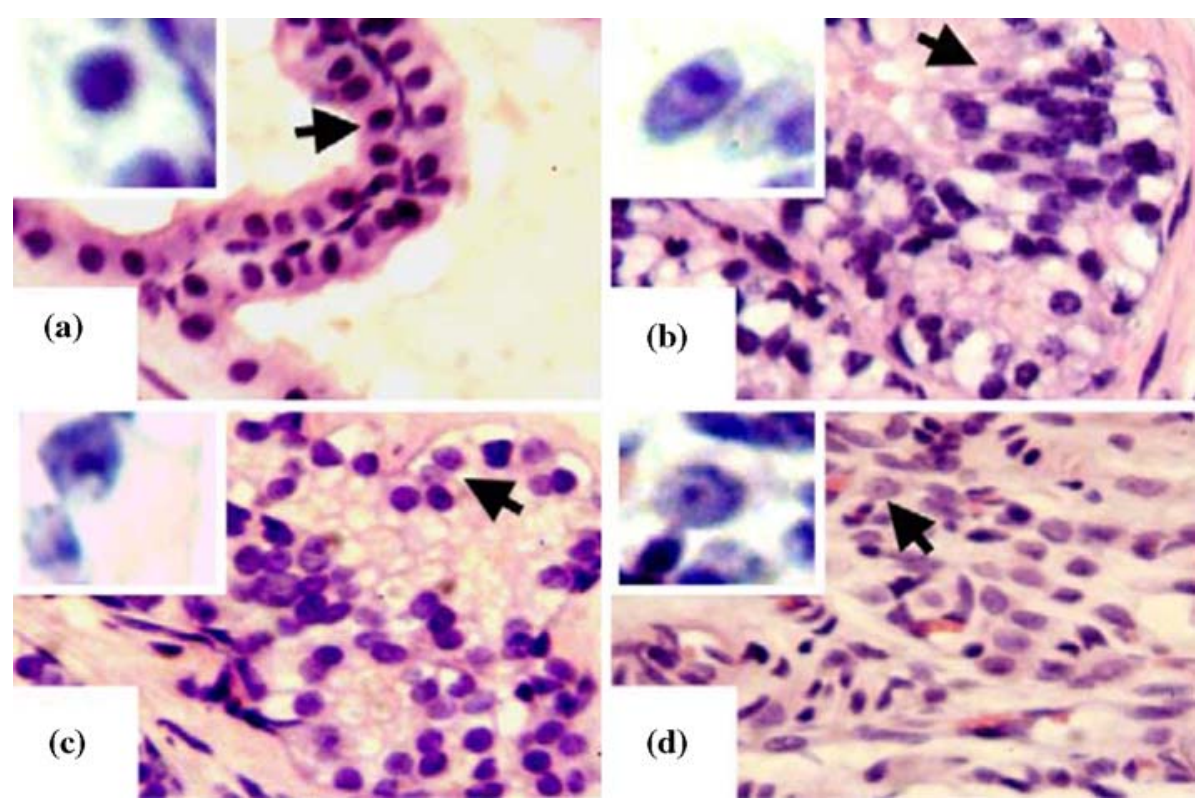

Figure 3. Cytological effects of Hedgehog overexpression at 30 days after the injection as shown by Hematoxylin-Eosin stain. The arrow-indicated cells were further magnified and presented in the inlets to show cytological changes of the nuclei and the nucleoli. (a) A prostate with $0.9 \% \mathrm{NaCl}$ injection showed no tumorigenic characteristics. (b) A pCX-shh-IG-injected prostate exhibited PIN formation with cells containing enlarged nuclei and prominent nucleoli. (c) A pCX-shh-IG-injected prostate exhibited the same PIN characteristics as in (b), but with smaller glands and loss of basal cell layer surrounding the arrow-indicated area, suggestive of $\mathrm{CaP}$ formation. (d) Mix-up of invasive $\mathrm{CaP}$ cells with stromal cells. Magnifications: $400 \times$ for (a) to (d).

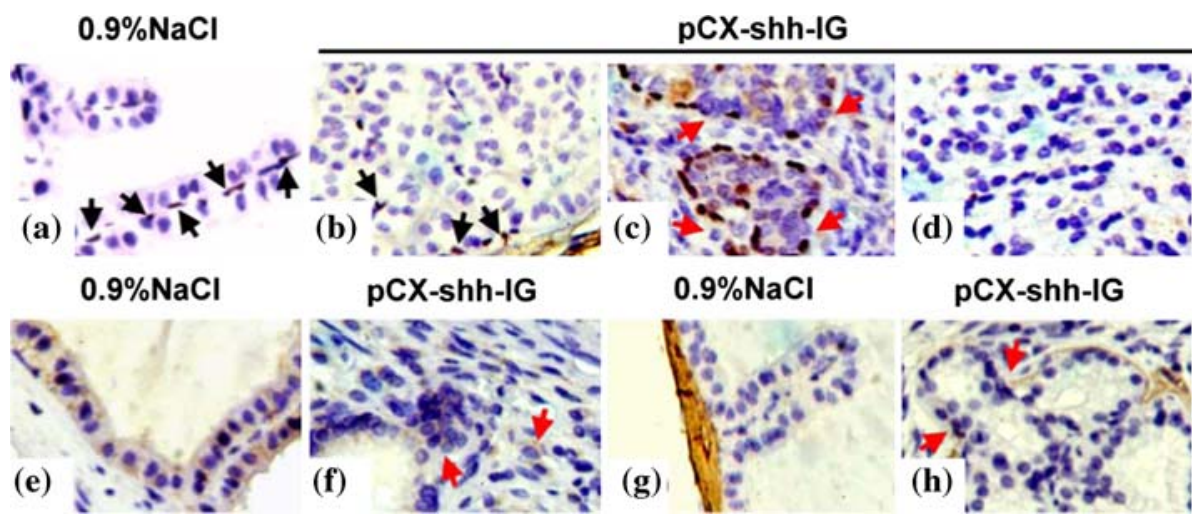

Figure 4. Immunohistochemical detections of p63 (basal cell marker), $\alpha$-SMA (fibro-muscular cell marker), and CK-8/CK-18 (epithelial cell marker) for further confirmation of tumorigenic phenotypes induced by Hedgehog overexpression. (a-d) Were from p63 detections; (e) and (f) from CK-8/CK-18 detections; (g) and (h) from $\alpha$-SMA detections. (a, e and g) Were from $0.9 \% \mathrm{NaCl}$-injected prostates; ( $b-d, f, h)$ Were from pCX-shh-IG-injected prostates. (a) p63 positive cells (arrow-indicated) showed a normal continuous layer of basal cells. (b) A discontinuous distribution of basal cell layer (arrow-indicated), indicative of PIN formation. (c) A smaller gland, with atypical cells filled the lumen of the duct and loss of p63 positive cells in some foci (arrow-indicated), indicative of invasive $\mathrm{CaP}$ formation. (d) A CaP formation with loss of p63 positive cells and absence of fibro-muscular sheath. (e) A normal CK-8/CK-18 positive epithelial distribution. (f) A prostate showed mix-up of epithelial cells in the stroma (arrow-indicated), indicative of invasive CaP formation. (g) A normal thick, dense, and continuous $\alpha$-SMA positive fibro-muscular sheath. (h) A thin, discontinuous $\alpha$-SMA positive fibro-muscular sheath following pCX-shh-IG injection. The arrows indicate the sites of discontinuity. Magnifications: $400 \times$ for (a) to (h). 


\section{Discussion}

Despite previous reports regarding involvement of Hedgehog protein in the cancer formation within sites of epithelial-mesenchymal interactions, there has been no report to confirm that Hedgehog can actually induce prostate cancer. In the present report, we have shown that Hedgehog overexpression resulted in prostate tumorigenesis, starting from the normal condition in the adult mouse prostates. We further characterized and compared the morphological aspects of $\mathrm{CaP}$ formation between the mouse specimen driven by Hedgehog overexpression and the human $\mathrm{CaP}$ specimen that had been diagnosed by clinical pathologists. Our results showed comparable morphological characteristics. Furthermore, we demonstrated Hedgehog activation in the human $\mathrm{CaP}$ specimen, in concordance with the Hedgehog overexpression in the mouse model. Moreover, we did not find comparable PIN formation in the pCX-IG nor the $0.9 \%$ $\mathrm{NaCl}$ injections, indicating that the PIN or CaP formation in the pCX-shh-IG group was not likely due to acute inflammatory response following the injection or the electroporation procedures. To our knowledge, this is the first data showing Hedgehoginduced in vivo prostate tumorigenesis that is initiated from the normal status.

Noticeably, we demonstrated that Hedgehog overexpression caused PIN formation within 30 days after the procedure. The PIN formation was faster than any other rodent model that had been reported to transform normal prostate epithelium into neoplasia under in vivo conditions. To confirm such fast effect caused by Hedgehog overexpression, we examined the presence of GFP as the marker of functional Hedgehog protein in the pCX-shh-IG injections. We found that PIN and CaP formation was only present in the pCX-shh-IG injection group, supporting the specific effects of Hedgehog protein during prostate tumorigenesis. Despite that the progression of PIN and even CaP formation in only 30 days was much faster than any previous model, this dramatically accelerated tumor growth had also been found in a xenograft model recently published by Fan et al. [22], supporting the potent effect through Hedgehog overexpression. Another line of evidence to support the fast $\mathrm{CaP}$ growth in the present model was the concomitant acceleration of CaP metastatic activity, since elevated Hedgehog signaling has also been implicated in metastasis [23]. With Hedgehog overexpression, we have been able to locate metastatic lesions in supra-epididymal lymph nodes along with upregulated expression of Twist (Chen et al., unpublished data), a major metastatic factor that has recently been implicated in prostate cancer malignancy [29, 30].

Further comparison with the xenograft model by Fan et al. [22] revealed differences in some aspects. The xenograft model did not show an increase in stromal proliferation, very much in contrast to the commonly found stromal hyperplasia in our model. This deviation could be due to different overexpression sites achieved by subcutaneous injections [22] vs. intra-prostatic injections (present study). Another possibility could be the difference in Hedgehog expression levels. A third potential cause of the deviation could be the differential intrinsic characteristics in interpreting Hedgehog signaling between the already malignant LNCaP tumor cells and the normal epithelial cells. Furthermore, it should be noted that in this intra-prostatic injection model Hedgehog protein was found highly expressed in the stromal compartment, which was likely to produce a condition not normally seen in other models of mouse prostate tumorigenesis. Evidently, these deviations and further characterization of Hedgehog-induced malignancy warrant subsequent investigations.

The molecular mechanisms underlying prostate tumorigenesis remain largely unknown. Although many recently identified genes have been implicated in the progression of prostate cancer, relatively few were suggested to initiate prostate tumorigenesis. On the epithelial side, loss of function of some genes, NKx 3.1 and Pten for example [31], were shown to cause hyperplasia and dysplasia of epithelial cells that are supposed to be "basal stem cells" [32] in mouse models. The loss-of-function was thought to progress, with accumulation of lesions in $\mathrm{Rb}$ and p53 tumor suppressor genes, from benign androgen-dependent hyperplasia to aggressive androgen-independent carcinoma [31]. On the stromal side, evidence has been accumulated to indicate a "reactive stroma" that provides secretory factors that contribute to tumor growth and progression [33]. It has been shown that dedifferentiation of stromal smooth muscle [34] and distortion of epithelial cell lineage proportions [32] are factors in prostate carcinogenesis. Indeed, there are factors that 
involve in both epithelial and stromal cells, with activities shifted between both sides or between different forms of a same factor, in correlation with progression from normal to malignant stages [35-38]. In the present study, we have demonstrated that Hedgehog overpression caused prostate tumorigenesis from normal status to $\mathrm{CaP}$ formation, and such transformation involved morphological characteristics within both the epithelial and the stromal sides. We also clearly showed that Hedgehog protein was detected within both the epithelial and the stromal cells during tumorigenesis, with concomitant loss of fibromuscular layer. Clearly, how does Hedgehog protein interact with presently known factors from both sides will attract immediate attention in human prostate cancer research.

In recent years, a variety of Hedgehog signaling inhibitors have been under development to act as potential cures for tumors caused by Hedgehog dysregulation [39]. Extensive use of animal models will be mandatory to test the efficacy of new therapeutic agents. Ideally, prostate cancer animal models should present as many as possible the human pathological features and simultaneously reflect the underlying molecular pathogenesis. Several types of mouse models have been established, namely reconstitution models, xenograft models, hormonal models, as well as transgenic and knockout models [31, 40]. However, various deviations have been observed in the mouse models. For example, the rat probasin promoter was used to drive the expression of SV40 large T and small $t$ antigens, producing the TRAMP (transgenic adenocarcinoma mouse prostate) prostate cancer model or, if without the small $t$ antigen, the LADY model $[40,41]$. These transgenic models, also called SV40-Tag models [26], displayed neuroendocrine features that are only seen in about $10 \%$ of human cases [42]. Another example of deviation is seen in some mouse transgenic or knockout models produced without using the SV40-Tag [3]. The nonSV40-Tag models displayed high proportions of atypical epithelial lesions representing different degrees of PIN without frequent progression into invasive carcinoma, making it difficult to prove their malignant potential [26]. These deviations do not necessarily devaluate the usage of mouse models, but reflect the complexity of mammalian prostate tumorigenesis and, at the same time, a demand for more animal models.
Another potential obstacle in using the established transgenic models is that whether Hedgehog signaling is dysregulated therein has not yet profiled. For this consideration, it would be straightforward to establish transgenic mouse model with in vivo Hedgehog overexpression. However, this is not always achievable as transgenic mice overexpressing Shh do not survive to stages when prostate cancer could be induced (Anthony E. Oro, personal communication). Until any conditional Hedgehog overexpression model becomes available, we believe that the present model, with its high efficiency of production and fast progression from benign to malignant stages and with major molecular events characterized, will facilitate therapeutic drug testing as well as basic prostate cancer research.

\section{References}

1. Greenlee R.T., Murray T., Bolden S. and Wingo P.A., Cancer statistics, 2000. CA Cancer J. Clin. 50: 7-33, 2000.

2. Isaacs W., De Marzo A. and Nelson W.G., Focus on prostate cancer. Cancer Cell 2: 113-116, 2000.

3. Abate-Shen C. and Shen M.M., Mouse models of prostate carcinogenesis. Trends Genet. 18: S1-S5, 2000.

4. De Marzo A.M., Meeker A.K., Zha S., Luo J., Nakayama M., Platz E.A., Isaacs W.B. and Nelson W.G., Human prostate cancer precursors and pathobiology. Urology 62: 55-62, 2003.

5. Huss W.J., Maddison L.A. and Greenberg N.M., Autochthonous mouse models for prostate cancer: past, present and future. Semin. Cancer Biol. 11: 245-260, 2001.

6. Ingham P.W. and McMahon A.P., Hedgehog signaling in animal development: paradigms and principles. Genes Dev. 15: 3059-3087, 2001.

7. McMahon A.P., Ingham P.W. and Tabin C.J., Developmental roles and clinical significance of hedgehog signaling. Curr. Top. Dev. Biol. 53: 1-114, 2003.

8. Podlasek C.A., Barnett D.H., Clemens J.Q., Bak P.M. and Bushman W., Prostate development requires Sonic hedgehog expressed by the urogenital sinus epithelium. Dev. Biol. 209: 28-39, 1999.

9. Lamm M.L., Catbagan W.S., Laciak R.J., Barnett D.H., Hebner C.M., Gaffield W., Walterhouse D., Iannaccone P. and Bushman W., Sonic hedgehog activates mesenchymal Glil expression during prostate ductal bud formation. Dev. Biol. 249: 349-366, 2002.

10. Freestone S.H., Marker P., Grace O.C., Tomlinson D.C., Cunha G.R., Harnden P. and Thomson A.A., Sonic hedgehog regulates prostatic growth and epithelial differentiation. Dev. Biol. 264: 352-362, 2003.

11. Berman D.M., Desai N., Wang X., Karhadkar S.S., Reynon M., Abate-Shen C., Beachy P.A. and Shen M.M., Roles for Hedgehog signaling in androgen production and prostate ductal morphogenesis. Dev. Biol. 267: 387-398, 2004. 
12. Watkins D.N., Berman D.M., Burkholder S.G., Wang B., Beachy P.A. and Baylin S.B., Hedgehog signalling within airway epithelial progenitors and in small-cell lung cancer. Nature 422: 313-317, 2003.

13. Thayer S.P., di Magliano M.P., Heiser P.W., Nielsen C.M., Roberts D.J., Lauwers G.Y., Qi Y.P., Gysin S., Fernandezdel Castillo C., Yajnik V., Antoniu B., McMahon M., Warshaw A.L. and Hebrok M., Hedgehog is an early and late mediator of pancreatic cancer tumorigenesis. Nature 425: 851-856, 2003.

14. Berman D.M., Karhadkar S.S., Maitra A., Montes De Oca R., Gerstenblith M.R., Briggs K., Parker A.R., Shimada Y., Eshleman J.R., Watkins D.N. and Beachy P.A., Widespread requirement for Hedgehog ligand stimulation in growth of digestive tract tumours. Nature 425: 846-851, 2003.

15. Kumamoto H., Ohki K. and Ooya K., Expression of Sonic hedgehog ( $\mathrm{SHH})$ signaling molecules in ameloblastomas. J. Oral Pathol. Med. 33: 185-190, 2004.

16. Goodrich L.V., Milenkovic L., Higgins K.M. and Scott M.P., Altered neural cell fates and medulloblastoma in mouse patched mutants. Science 277: 1109-1113, 1997.

17. Oro A.E., Higgins K.M., Hu Z., Bonifas J.M., Epstein E.H. Jr. and Scott M.P., Basal cell carcinomas in mice overexpressing sonic hedgehog. Science 276: 817-821, 1997.

18. Aboulkassim T.O., LaRue H., Lemieux P., Rousseau F. and Fradet Y., Alteration of the PATCHED locus in superficial bladder cancer. Oncogene 22: 2967-2971, 2003.

19. Dahmane N., Sanchez P., Gitton Y., Palma V., Sun T., Beyna M., Weiner H. and Ruiz i Altaba A., The Sonic Hedgehog-Gli pathway regulates dorsal brain growth and tumorigenesis. Development 128: 5201-5212, 2001.

20. Sanchez P., Hernandez A.M., Stecca B., Kahler A.J., DeGueme A.M., Barrett A., Beyna M., Datta M.W., Datta S. and Ruizi Altaba A., Inhibition of prostate cancer proliferation by interference with SONIC HEDGEHOGGLI1 signaling. Proc. Natl. Acad. Sci. USA 101: 1256112566, 2004

21. Sheng T., Li C., Zhang X., Chi S., He N., Chen K., McCormick F., Gatalica Z. and Xie J., Activation of the hedgehog pathway in advanced prostate cancer. Mol. Cancer 3: 29, 2004

22. Fan L., Pepicelli C.V., Dibble C.C., Catbagan W., Zarycki J.L., Laciak R., Gipp J., Shaw A., Lamm M.L., Munoz A., Lipinski R., Thrasher J.B. and Bushman W., Hedgehog signaling promotes prostate xenograft tumor growth. Endocrinology 145: 3961-3970, 2004.

23. Karhadkar S.S., Bova G.S., Abdallah N., Dhara S., Gardner D., Maitra A., Isaacs J.T., Berman D.M. and Beachy P.A., Hedgehog signalling in prostate regeneration, neoplasia and metastasis. Nature 431: 707-712, 2004.

24. Oberg K.C., Pira C.U., Revelli J.P., Ratz B., AguilarCordova E. and Eichele G., Efficient ectopic gene expression targeting chick mesoderm. Dev. Dyn. 224: 291-302, 2002.

25. Nakamura $H$. and Funahashi J., Introduction of DNA into chick embryos by in ovo electroporation. Methods 24: 4348, 2001.

26. Park J.H., Walls J.E., Galvez J.J., Kim M., Abate-Shen C., Shen M.M. and Cardiff R.D., Prostatic intraepithelial neoplasia in genetically engineered mice. Am. J. Pathol. 161: 727-735, 2002.

27. Peterson G.L., A simplification of the protein assay method of Lowry et al. which is more generally applicable. Anal. Biochem. 83: 346-356, 1997.
28. Laemmli U.K., Cleavage of structural proteins during the assembly of the head of bacteriophage T4. Nature 227: 680-685, 1970.

29. Yang J., Mani S.A., Donaher J.L., Ramaswamy S., Itzykson R.A., Come C., Savagner P., Gitelman I., Richardson A. and Weinberg R.A., Twist, a master regulator of morphogenesis, plays an essential role in tumor metastasis. Cell 117: 927-939, 2004.

30. Kwok W.K., Ling M.T., Lee T.W., Lau T.C., Zhou C., Zhang X., Chua C.W., Chan K.W., Chan F.L., Glackin C., Wong Y.C. and Wang X., Up-regulation of TWIST in prostate cancer and its implication as a therapeutic target. Cancer Res. 65: 5153-5162, 2005.

31. Abate-Shen C. and Shen M.M., Molecular genetics of prostate cancer. Genes Dev. 14: 2410-2434, 2000.

32. van Leenders G.J. and Schalken J.A., Epithelial cell differentiation in the human prostate epithelium: implications for the pathogenesis and therapy of prostate cancer. Crit. Rev. Oncol. Hematol. 46: S3-S10, 2003.

33. Sung S.Y. and Chung L.W., Prostate tumor-stroma interaction: molecular mechanisms and opportunities for therapeutic targeting. Differentiation 70: 506-521, 2002.

34. Wong Y.C. and Tam N.N., Dedifferentiation of stromal smooth muscle as a factor in prostate carcinogenesis. Differentiation 70: 633-645, 2002.

35. Fudge K., Bostwick D.G. and Stearns M.E., Plateletderived growth factor $\mathrm{A}$ and $\mathrm{B}$ chains and the alpha and beta receptors in prostatic intraepithelial neoplasia. Prostate 29: 282-286, 1996.

36. Kaplan P.J., Mohan S., Cohen P., Foster B.A. and Greenberg N.M., The insulin-like growth factor axis and prostate cancer: lessons from the transgenic adenocarcinoma of mouse prostate (TRAMP) model. Cancer Res. 59: 2203-2209, 1999.

37. Jin C., McKeehan K., Guo W., Jauma S., Ittmann M.M., Foster B., Greenberg N.M., McKeehan W.L. and Wang F., Cooperation between ectopic FGFR1 and depression of FGFR2 in induction of prostatic intraepithelial neoplasia in the mouse prostate. Cancer Res. 63: 8784-8790, 2003.

38. Evangelou A.I., Winter S.F., Huss W.J., Bok R.A. and Greenberg N.M., Steroid hormones, polypeptide growth factors, hormone refractory prostate cancer, and the neuroendocrine phenotype. J. Cell. Biochem. 91: 671-683, 2004.

39. Rice R., Spencer-Dene B., Connor E.C., Gritli-Linde A., McMahon A.P., Dickson C., Thesleff I. and Rice D.P., Disruption of Fgf10/Fgfr2b-coordinated epithelial-mesenchymal interactions causes cleft palate. J. Clin. Invest. 113 . 1692-1700, 2004.

40. Navone N.M., Logothetis C.J., von Eschenbach A.C. and Troncoso P., Model systems of prostate cancer: uses and limitations. Cancer Metast. Rev. 17: 361-371, 1998.

41. Huss W.J., Hanrahan C.F., Barrios R.J., Simons J.W. and Greenberg N.M., Angiogenesis and prostate cancer: identification of a molecular progression switch. Cancer Res. 61: 2736-2743, 2001.

42. Masumori N., Tsuchiya K., Tu W.H., Lee C., Kasper S., Tsukamoto T., Shappell S.B. and Matusik R.J., An allograft model of androgen independent prostatic neuroendocrine carcinoma derived from a large probasin promoter-T antigen transgenic mouse line. J. Urol. 171: 439-442, 2004 